\title{
Probing plasmonic system by the simultaneous measurement of Raman and fluorescence signals of dye molecules
}

\author{
M.M. Dvoynenko ${ }^{1}$, Z.I. Kazantseva ${ }^{1}$, V.V. Strelchuk ${ }^{1}$, O.F. Kolomys ${ }^{1}$, E.G. Bortshagovsky ${ }^{1}$, \\ E.F. Venger ${ }^{1}, \mathbf{P}$. Tronc ${ }^{2}$ \\ ${ }^{1}$ V. Lashkaryov Institute of Semiconductor Physics, National Academy of Sciences of Ukraine, \\ 41, prosp. Nauky, 03028 Kyiv, Ukraine \\ ${ }^{2}$ Centre National de la Recherche Scientifique, Ecole Superieure de Physique et de Chimie Industrielles de la Ville de \\ Paris, 10 rue Vauquelin, 75005 Paris, France
}

\begin{abstract}
The simultaneous measurement of Raman and fluorescence signals was proposed to find out the molecule-metal distance. The ratio between Raman and fluorescence intensities was used to estimate molecule-metal distance in nanometer scale. A low-value intensity of the fluorescence of the dye molecules was found using the photobleaching effect. Made was a comparison of experimental results with a theoretical model, which showed well agreement.
\end{abstract}

Keywords: SERS, photobleaching effect, fluorescence, dye molecule, Au surface.

Manuscript received 14.10.10; accepted for publication 16.03.11; published online 30.06.11.

\section{Introduction}

There is a great interest to surface plasmon and related phenomena [1]. Surface and tip enhanced Raman spectroscopies (SERS and TERS) [2], surface plasmon enhanced light emission [3-5] are in a researchers' focus of interest. The intensity of surface-enhanced fluorescence and SERS depends on the local field and energy transfer from an excited molecule to a metal particle, which depends on the distance between the molecule and metal particle. Thus, to control the molecule-metal distance is of great importance to describe the plasmon system. Dependence of the fluorescence lifetime of dyes on the molecule-metal distance was studied at wavelength [6]- and nm [7]-scale distances. The molecule - single nanoparticle distance dependence of both fluorescence [8,9] and TERS [10] intensities was experimentally demonstrated. However, separate fluorescence and Raman studies in experiments with many molecules have a problem associated with various molecule numbers. Additionally, the number of molecules situated in light spot during the measurement can be decreased due to photobleaching. Since the photobleaching time depends on the moleculenanoparticle distance, the distance behaviors of the Raman and fluorescence intensities will be changed for different accumulation times. Simultaneous Raman and fluorescence measurements [11] could resolve this general problem. Indeed, on the one hand, both Raman and fluorescence intensities are determined by the same studying molecule' numbers, on the other hand, there is a difference between them. Both Raman and fluorescence intensities depend on the electromagnetic enhancement factor [11-13], while the fluorescence intensity depends also on the decay rate [11-13] for molecules, which just reflects the energy transfer from the excited molecule to metal. As a consequence, the ratio between simultaneously measured Raman and fluorescence intensities, cancelling the associated molecule quantity and electromagnetic enhancement factor, is only a function of the decay rate of these molecules that in its turn is strongly sensitive to the molecule-metal distance. The simultaneous Raman and fluorescence measurement was proposed [11] as a way to find the chemical enhancement factor. In the absence of the chemical enhancement it can be used [14] to find the molecular lifetime in steady-state spectroscopy without resorting to time-resolved spectroscopy. Note that the simultaneous observation of Raman and fluorescence signals was reported [15] but there was no analysis or proposals to investigate the distance dependence. Recently [16], the simultaneous measurement of the Raman and fluorescence intensities and an analysis of the molecule-metal distance dependence were done for dye molecules of rhodamine $6 \mathrm{G}$. In this paper, we experimentally study the distance behavior of the ratio between Raman and fluorescence intensities for dye molecules of 2-(4-(p-dimethylaminophenyl)-1,3-butadienyl)-3-ethylbenzothoazolium perchlorat (LDS 721 or styryl 8). 


\section{Experiment}

In this paper, we experimentally demonstrate and analyze the distance dependence of the ratio between Raman and fluorescence intensities of dye molecules of LDS 751 spaced by a thin polymer film at nm-scale distance at a gold substrate. A smooth flat metal surface is chosen because all measured molecules are approximately at the same distance to the metal surface in contrast to island metal films [15]. A smooth gold film with the thickness about $100 \mathrm{~nm}$ was prepared by thermal evaporation on a glass substrate. Monolayers prepared using polymer (polyimide) solution in chloroform were investigated by R\&K (Wiesbaden, Germany) equipment for Langmuir-Blodgett film (LBF) preparation. LBFs [17] were formed in the standard way of vertical transfer under control of the transfer coefficient and $\pi$-A - diagrams. The LBFs were deposited at the surface tension of $20 \mathrm{mN} / \mathrm{m}$ by upstrokes to form Z-type structure on the substrates. The dipping rate was $2 \mathrm{~mm} / \mathrm{min}$. The LBF thickness was measured ellipsometrically using a laser beam $\left(\lambda=0.623 \mu \mathrm{m} ;\right.$ incidence angle $\left.60^{\circ}\right)$. The reversed ellipsometric problem was solved numerically for the "substrate-optically transparent film" model. Ellipsometric measurements with a ten-layer film give both refractive index 1.64 and thickness $0.85 \pm 0.15 \mathrm{~nm}$ per layer. The refractive index 1.64 is comparable to that obtained previously [18]. LDS 751 molecules were deposited by the dipping with the speed $1 \mathrm{~mm} / \mathrm{s}$ in water solution of the dye with the concentration of $10^{-5} \mathrm{M}$. The confocal micro-Raman and fluorescence spectra were measured by the T64000 Jobin Yvons spectrometer with a thermoelectrically cooled charge coupled device detector. The 514.5-nm line of an $\mathrm{Ar}^{+} / \mathrm{Kr}^{+}$-ion laser was used as the excitation source. After being reflected from a sharp-edge Raman filter, the laser beam was then focused by an Olympus BX41 microscope equipped with an objective $\times 100$ (numerical aperture $(\mathrm{NA})=0.90$ ) on the sample surface. The net laser intensity on the sample surface was $\sim 5 \times 10^{7} \mathrm{~W} / \mathrm{m}^{2}$. The light scattered backward was collected through the same objective and detected with the integration time $20 \mathrm{~s}$. All spectra were measured at the room temperature $(T=300 \mathrm{~K})$.

\section{Results and discussion}

Due to the photobleaching effect, the studied frequency region $\left(500-2500 \mathrm{~cm}^{-1}\right)$ was chosen in such a way that a spectrum was measured without rotating the monochromator grating. On the other hand, this frequency range had a maximal value in order to compare various active Raman modes of LDS 751 molecules. The Raman and fluorescence spectra of LDS 751 deposited on a gold substrate with the accumulation time $300 \mathrm{~s}$ are shown in Fig. 1.The most pronounced Raman peaks are at 1130,1280 and $1564 \mathrm{~cm}^{-1}$. The peak at $1564 \mathrm{~cm}^{-1}$ with the maximal magnitude is in focus of our study. The fluorescence spectrum of LDS 751 is broad with the peak at approximately $751 \mathrm{~nm}$. The Raman and fluorescence spectra at the spacer thicknesses 0.85, 2.55 and $4.25 \mathrm{~nm}$ are shown in Fig. 2, which correspond to the number $N=1,3,5$ of the polyimide monolayers. At the first sight, it can seem that the Raman intensity decreases with the thickness of the spacer. The local field near a flat surface consists of incident and reflected fields. At very small thicknesses (few nanometers) of the spacer, the reflected field can be changed insignificantly. It is likely that the surface to be not enough smooth and the electromagnetic enhancement factor decreases with the distance to some inhomogeneity (the enhancement factor practically does not depend at such nm-scale distances to the surface for ideal plane surface). However, the main reason in the decrease of the Raman intensity with the distance consists in the photobleaching effect during the measurement. As the photobleaching time being a result of a competition between the energy transfer from excited molecule to the metal and relaxation to a triplet state [19] strongly decreases with the spacer thickness, the average number of measured molecules decreases with the distance to metal surface, too.

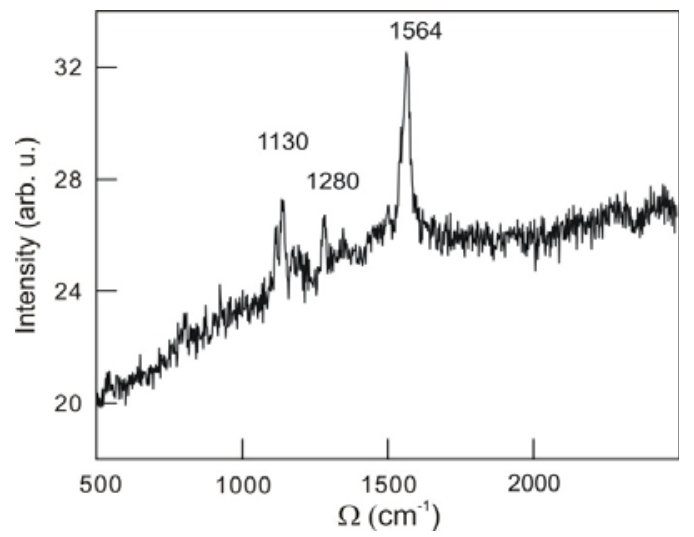

Fig. 1. The Raman and fluorescence spectra of LDS 751 deposited on a gold substrate.

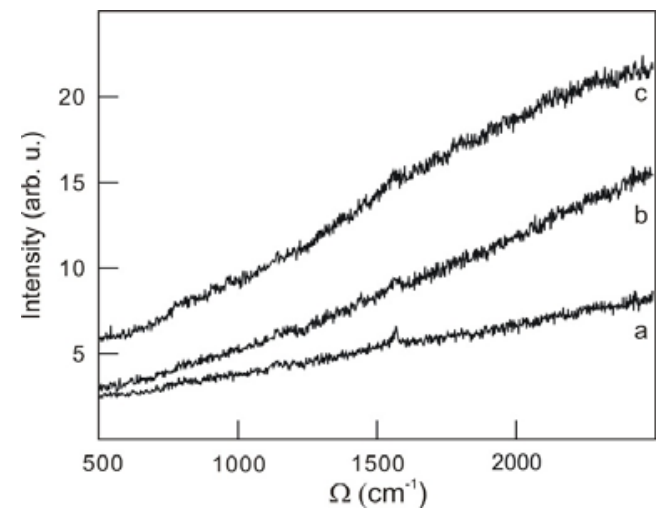

Fig. 2. The confocal micro-Raman and fluorescence intensities of the LDS 751 on the Raman shift $\Omega$. The characters (a, b, c) indicate curves with the numbers $N=1,3$ and 5 of the polyimide monolayers, respectively. 
Contrary to the Raman signal, the fluorescence intensity cannot be found directly from the data in Fig. 2 due to a small value of the signal and the absence of the information about zero level at the wing of the Rayleigh line. In order to resolve this problem, we used the photobleaching effect. Spectra of LDS 751 at $N=1$ obtained for various illumination times before measurements are presented in Fig. 3. The subtraction between the different spectra gives the value of the Raman and fluorescence intensities of molecules that were photo-oxidized. For example, the subtractions $I_{a}-I_{b}$ between the curves $a$ and $b$ and $I_{b}-I_{c}$ between the curves $b$ and $c$ in Fig. 3 give the correct (without background) Raman and fluorescence intensities of the molecules that were photo-oxidized during the illumination times 20 and $80 \mathrm{~s}$, respectively. By this way, the background level was cancelled and the ratio between Raman and fluorescence intensities $I_{R} / I_{f l}$ was found for the Raman shifts $\Omega=1564 \mathrm{~cm}^{-1}$ for all spacer thicknesses.

Typically, $I_{R} / I_{f l}$ little increases with the illumination time. It can be simply explained. Indeed, in the absence of the chemical enhancement $I_{R} / I_{f l} \sim \gamma$ [11], where $\gamma$ is the decay rate of the molecule that is the value to be inversed to the lifetime of the excited molecule. The higher lifetime of an excited molecule leads to a higher probability to be situated in a metastable triplet energy state and consequently to transfer of the energy to oxygen. Thus, the photo-oxidation of the molecules having the higher lifetime has the higher probability. Since in the electrostatic approximation and in the absence of the physical spacer layer $\gamma \sim z^{-3}$ ( $z$ is the molecule-surface distance) for the case of the volume damping [20], one can say that the photobleaching is able to selectively "delete" the most remote dye molecules. One more trendlike property is an increase of the fluctuation of $I_{R} / I_{f l}$ with the spacer thickness decrease. The highest fluctuations are at $N=1$. This property is clearly seen taking into account the distance dependence of the decay rate. Indeed, $\left|\frac{\Delta \gamma}{\gamma}\right|=3\left|\frac{\Delta z}{z}\right|$ for the case of the volume damping. Thus, the fluctuation of the molecule-surface distance essentially increases at small mean distances. If there is the spacer layer and the distance between the molecule and surface of the spacer is not equal to zero, then we obtain even in the electrostatic

approximation $\gamma \sim\left(\frac{A_{1}}{(z+h)^{3}}+\frac{A_{2}}{(z+2 h)^{3}}+\frac{A_{3}}{(z+3 h)^{3}}+\ldots\right)$,

where

$A_{1}, A_{2}, A_{3}, \ldots$ are some coefficients. The latter expression represents multiple reflections in the spacer film. Note that the multiple reflections still keeps the general feature about an increase of the fluctuations at smaller spacer thicknesses.

The main result of the molecule-surface distance behavior is depicted in Fig. 4. The diapasons of the values $I_{R} / I_{f l}$ for the Raman shift $\Omega=1564 \mathrm{~cm}^{-1}$ are presented as vertical lines at the five number values of the spacer layers. It is seen that the fluctuation decreases with the spacer thickness. Theoretical calculation is presented by the solid line supposing that $I_{R} / I_{f l}=1.23$ at $N=1$. This calculation is made at the following parameters: the distance of the point-like source to surface should be $1 \mathrm{~nm}$ and the thickness of one monolayer should be $0.85 \mathrm{~nm}$. The dielectric function $\varepsilon$ of the polyimide spacer is taken as $\varepsilon=2.8$. Theoretical calculation of the non-radiative relaxation rate based on the classical theory delineated in Ref. [20] was performed. Briefly, the relaxation rate of a dipole, $\boldsymbol{p}$, placed near a metallic surface is given by $\gamma \propto \operatorname{Im}\left(\boldsymbol{p}^{*} \cdot \boldsymbol{E}_{R}\right)$, where $\boldsymbol{E}_{R}$ is the reaction field containing direct incident (radiated) field and reflected field from the surface. From the latter equation, one can get $\gamma \propto|p|^{2} \operatorname{Im}\left(G_{i i}(\omega, \vec{r}, \vec{r})\right)$, where $\left(G_{i i}(\omega, \vec{r}, \vec{r})\right)$ is the corresponding component of the electrodynamics Green tensor. In the case of a substrate with the spacer layer, the Green function can be found elsewhere [21]. Supposing the case of the physical adsorbed molecule

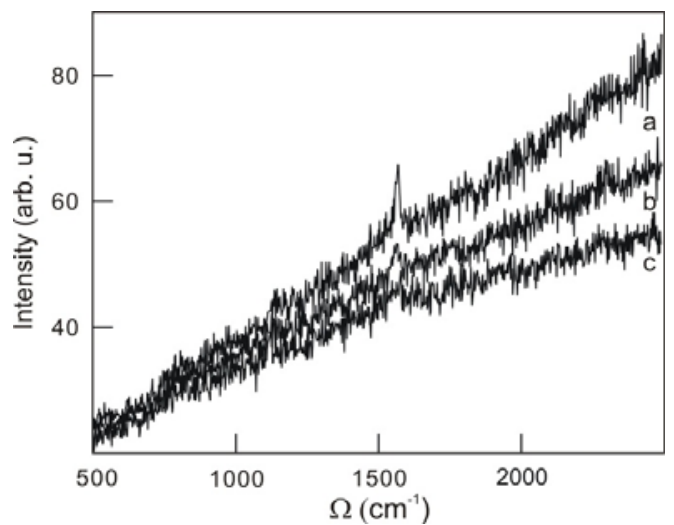

Fig. 3. The confocal micro-Raman and fluorescence spectra at $N=1$ measured after preliminary light illumination at the operation wavelength and intensity for duration $0(a), 20(b)$, and $80 \mathrm{~s}(c)$.

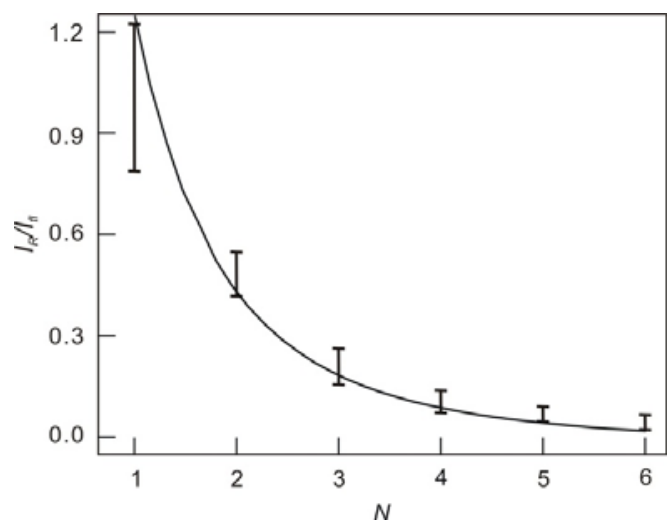

Fig. 4. Experimental (vertical lines) and theoretical (solid curve) dependences of $I_{R} / I_{f l}$ on the monolayer number $N$. 
(the dipole moment does not change), one can get $I_{R} / I_{f l} \sim \operatorname{Im}\left(G_{i i}(\omega, \vec{r}, \vec{r})\right)$. This expression is presented in Fig. 4 by the solid line. Of course, the coefficient in the equation $I_{R} / I_{f l} \sim \operatorname{Im}\left(G_{i i}(\omega, \vec{r}, \vec{r})\right)$ depends on many parameters, in particular, on the excitation and radiation wavelengths, kind of molecules, temperature, etc. However, it does not depend on the nanoplasmon system. Thus, finding once the coefficient at the fixed above mentioned parameters, one can make a diagnostic of the nanoplasmonic system only on the basis of the ratio between Raman and fluorescence intensities.

One can see that the theoretical dependence describes the experimental behavior quite well. However, there is a tendency for this behavior to be disagreed with theoretical dependence at small spacer thicknesses. A similar tendency [16] took place in the case of rhodamine $6 \mathrm{G}$ molecules. This tendency can be caused by both certain inhomogeneity in the spacer layer and the deposited dye film as well as by the presence of background. One can see that the value of the ratio $I_{R} / I_{f l}$ is less than the theoretical prediction. It looks like there is an additional fluorescence not taken into account. This fluorescence can be caused by an additional background. Nevertheless, though the subtraction should cancel the background associated with the fluorescence of $\mathrm{Au}$ and the wing of the Rayleigh scattering, an additional background can exist. Indeed, it has been reported recently [22] that non-fluorescent molecules (in our case dye molecules) can give some background. This background cannot be cancelled by the proposed subtraction procedure.

\section{Conclusion}

In conclusion, we have performed an experiment on simultaneous measurement of Raman and fluorescence intensities of molecules LDS 751 located at various distances from a flat $\mathrm{Au}$ surface. It was shown that the ratio between the Raman and fluorescence intensities is well described by the classical model of the energy transfer from the molecule to metal. We believe that this ratio can be used to find the distance between molecule and metal surface.

The authors are thankful to S.A. Zenyo for preparation of Au substrate film.

This work was supported by Ukrainian-French projects "Dnipro" of State fund for fundamental researches of Ukraine

\section{References}

1. W.L. Barnes, A. Dereux, and T.W. Ebbesen, Surface plasmon subwavelength optics // Nature, 424, p. 824-830 (2003).

2. For recent reviews, see: Surface-Enhanced Raman Scattering. Physics and Applications, edited by K. Kneip, M. Moskovits, and H. Kneip // Topics in Applied Physics, 103, p. 1-464 (2006).
3. K. Okamoto, I. Niki, A. Shvartser, Y. Narukava, T. Mukai, and A. Scherer, Surface-plasmonenhanced light emitters based on InGaN quantum wells // Nature Materials, 3, p. 601-605 (2004).

4. E. Fort and S. Gr'esillon, Surface-enhanced fluorescence // J. Phys. D: Appl. Phys.41, 013001013031 (2008).

5. J.R. Lakowitcz, Ch.D. Geddes, I. Gryczynski, J. Malicka, Z. Gryszynski, K. Aslan, J. Lukomska, E. Matveevs, J. Zhang, R. Badugu, and J. Huang, Advances in surface-enhanced fluorescence // J. Fluorescence, 14, p. 425-441 (2004).

6. K.H. Drexhage, Influence of a dielectric interface on fluorescence decay time // J. Luminescence, 1-2, p. 693-701 (1970).

7. G. Cnossen, K.E. Drabe, and D.A. Wiersma, Fluorescence properties of submonolayers of rhodamine $6 \mathrm{G}$ in front of a mirror // J. Chem. Phys., 98, p. 5276-5280 (1993).

8. P. Anger, P. Bgaradwaj, and L. Novotny, Enhancement and quenching of single-molecule fluorescence // Phys. Rev. Lett., 96, 113002-1 113002-4 (2006).

9. S. Kuhn, U. Hakanson, L. Rogobette, and V. Sandoghdar, Enhancement of single-molecule fluorescence using a gold nanoparticle as an optical nanoantenna // Phys. Rev. Lett., 97, 017402-1 017402-4 (2006).

10. A. Hartschuh, E.J. Sanchez, X.S. Xie, and L. Novotny, High-resolution near-field Raman microscopy of single-walled carbon nanotubes // Phys. Rev. Lett., 90, 095503-1 - 095503-4 (2003).

11. M.M. Dvoynenko and J.-K. Wang, Finding electromagnetic and chemical enhancement factors of surface-enhanced Raman scattering // Opt. Lett. 32, p. 3552-3554 (2007).

12. H. Xu, X-H. Wang, M.P. Persson, H.Q. Xu, M. Kall, and P. Johansson, Unified treatment of fluorescence and Raman scattering processes near metal surfaces // Phys. Rev. Lett., 93, 243002-1 243002-4 (2004).

13. P. Johansson, H. Xu, and M. Kall, Surfaceenhanced Raman scattering and fluorescence near metal nanoparticles // Phys. Rev. B, 72, 035427-1 035427-17 (2005).

14. C.M. Galloway, P.G. Etchegoin, and E.C. Le Ru, Ultrafast nonradiative decay rates on metallic surfaces by comparing surface-enhanced Raman and fluorescence signals of single molecules // Phys. Rev. Lett., 103, 063003-1 - 063003-4 (2009).

15. A.E. German and G.A. Gachko, Dependence of the amplification of giant Raman scattering and fluorescence on the distance between an adsorbed molecule and a metal surface // J. Appl. Spectr. 68, p. 987-992 (2001).

16. M.M. Dvoynenko, Z.I. Kazantseva, V.V. Strelchuk, O.F. Kolomys, E.F. Venger, and J.-K. Wang, Molecular ruler based on concurrent measurements 
of enhanced Raman scattering and fluorescence // Opt. Lett., 35, p. 3802-3804 (2010).

17. T. Akatsuka, H. Tanaka, J. Toyama, T. Nakamura, M. Matsumoto, and Y. Kawabata, Properties of liquid crystal cells of pyrolyzed polyimide Langmuir-Blodgett films // Thin Solid Films, 210/211, p. 458-460 (1992).

18. P.A. Kawka and R.O. Buckius, Optical properties of polyimide films in the infrared // Intern. J. Thermophys., 22, p. 517-534 (2001).

19. R. Zondervan, F. Kulzer, M.A. Kol'chenko, and M. Orrit, Photobleaching of rhodamine $6 \mathrm{G}$ in poly(vinyl alcohol) at the ensemble and singlemolecule levels // J. Phys. Chem. A, 108, p. 1657 1665 (2004).
20. R.R. Chance, A. Prock, and R. Silbey, Molecular fluorescence and energy transfer // Adv. Chem. Phys., 37, p. 1-65 (1978).

21. A.K. Zvezdin and V.I. Belotelov, Electrodynamic Green-function technique for investigating the magneto-optics of low-dimensional systems and nanostructures // J. Opt. Soc. Amer. B, 22, p. 228229 (2005).

22. S. Mahajan, R.M. Cole, J.D. Speed, S.H. Pelfrey, A.E. Russell, P.N. Bartlett, S.M. Barnett, and J.J. Baumberg, Understanding the surfaceenhanced Raman spectroscopy "background" // J. Phys. Chem. C, 114, p. $7242-7250$ (2010). 